NASATM- - 76- 207455

\title{
Numerical Solutions of Supersonic and Hypersonic Laminar Compression Corner Flows
}

C.M. Hung and R.W. MacCormack

NASA Ames Research Center, Moffett Field, Calif. 


$$
\bar{r}
$$




\title{
Numerical Solutions of Supersonic and Hypersonic Laminar Compression Corner Flows
}

\author{
C.M. Hung* and R.W. MacCormack $\dagger$ \\ NASA Ames Research Center, Moffett Field, Calif.
}

\begin{abstract}
An efficient lime-splitting, second-order accurate, numerical scheme is used to solve the complete NavierStokes equations for supersonic and hypersonic laminar flow over a two-dimensional compression corner. A fine, exponentially stretched mesh spacing is used in the region near the wall for resolving the viscous layer. Good agreement is obtained between the present computed results and experimental measurement for a Mach number of 14.1 and a Reynolds number of $1.04 \times 10^{5}$ with wedge angles of $15^{\circ}, 18^{\circ}$, and $24^{\circ}$. The details of the pressure variation across the boundary layer are given, and a correlation between the leading edge shock and the peaks in surface pressure and heat transfer is observed.
\end{abstract}

\section{Introduction}

C ONTINUING advances in numerical methods and computer capabilities have now made feasible many flowfield calculations which were formerly intractable. One such problem which has renewed interest is supersonic or hypersonic flow over a two-dimensional compression corner. This problem has received considerable attention within the past decade because of its importance to the design engineer in predicting the pressure and heat loads at a wing-flap junction on re-entry vehicles. When flow separation occurs, reduced flap effectiveness results, and in some regions, the surface heating could become severe on a maneuverable re-entry vehicle.

The problem to be considered is illustrated schematically in Fig. 1. The pressure rise generated by the wedge extends upstream along the flat plate, thickening the boundary layer, and results in a complicated interaction between the viscous flow near the body surface and the outer inviscid stream. Since the inner part of the boundary layer may not have sufficient momentum to overcome the combined effects of skin friction and adverse pressure gradient, the interaction can lead to flow separation for certain ranges of Mach number, Reynolds number, and wedge angle. The separated boundary layer will then become a free shear layer external to a recirculating inner flow near the corner. Reattachment occurs because of the interaction between free shear flow and the outer flow. The surface pressure continues to rise through the separated and reattached regions, until the boundary layer reaches a minimum thickness or "neck." Downstream of the neck, the boundary layer returns to a normal state of weak interaction with the outer inviscid stream at a new Mach number. Although, in most practical situations the region of shock wave and boundary-layer interaction is turbulent, at high altitude flight, fully laminar flows can exist and are important for design considerations.

Previous theoretical treatment of such a problem has usually been made with the boundary-layer equations together with a "coupling" equation relating the development of the inner viscous flow to the outer flow. The governing partial differential equations can then be solved by finite difference techniques ${ }^{1,2}$ or can be expressed as integral relations and

Presented as Paper 75-2 at the AIAA 13th Aerospace Sciences Meeting, Pasadena, California, January 20-22, 1975; submitted February 27, 1975; revision received July 7, 1975.

Index categories: Supersonic and Hypersonic Flow; Boundary Layers and Convective Heat Transfer-Laminar; Computer Technology and Computer Simulation Techniques.

*NRC Research Associate. Member AIAA.

†Assistant Chief, Computational Fluid Dynamics Branch. Member AIAA solved as ordinary differential equations. ${ }^{3-7}$ This treatment, in general, involves the question of uniqueness because certain portions of the flowfield contain substantial upstream influence, and the initial and downstream boundary conditions cannot be completely specified. In some integral techniques, ${ }^{4-7}$ there is also the question of so-called jump conditions for supercritical to subcritical types of boundary layers.

Consideration of the Navier-Stokes equations avoids these questions and removes some restrictive assumptions, viz., that the static pressure is constant across the boundary layer, and that the viscous and inviscid flows interact only along a line at or near the edge of the boundary layer, which can be difficult to define in hypersonic flow. Also, as mentioned by Van Dyke, ${ }^{8}$ a solution of the Navier-Stokes equations is necessary in the immediate vicinity of a sharp corner.

Carter ${ }^{9}$ has obtained numerical solutions of the NavierStokes equations for laminar flow past a compression corner at low Mach numbers. He used the Brailovskaya difference scheme, which is first-order accurate in time and second-order accurate in space. In the present study a more efficient numerical method, ${ }^{10,11}$ which is second-order accurate in both time and space, is used to calculate supersonic and hypersonic cases.

The complete time-dependent Navier-Stokes equations are split into two sets, one for the $x$-derivative and the other for the $y$-derivative. The advantage of the split system is that the computation proceeds with larger time increments because the stability criterion is less stringent. Each calculation starts from a uniform flow with appropriate boundary conditions. The boundary layer and shock waves generated by the leading edge and compression corner develop with time, until a steady state is achieved. A fine, exponentially stretched mesh spacing is employed in the region near the wall for resolving the viscous layer, and a coarse uniform mesh spacing is used in the outer region where viscous effects are negligible. The solution is advanced more frequently in the fine mesh region,

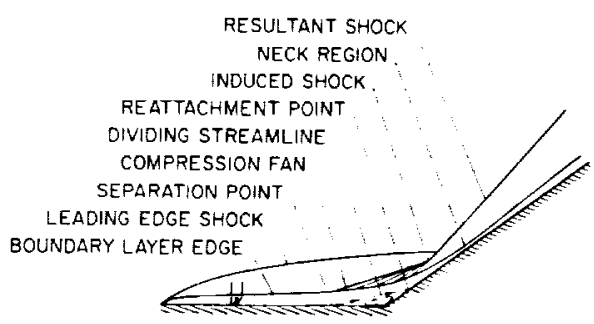

Fig. 1 Schematic diagram of supersonic flow over a sharp flat platewedge compression corner. 
using smaller time steps, than in the coarse mesh region. Computational efficiency is further enhanced by using elongated cells (nonisotropic mesh spacing).

A novel, recently devised fourth-order damping term is incorporated to maintain numerical stability in the presence of steep pressure gradients, and hence, fine mesh spacing near the leading edge, required by previous studies, is avoided. Moreover, high Mach number cases with strong induced shocks may be solved. The results are in good agreement with Carter's computed data, with significantly less computation time for a wedge angle of $10^{\circ}$, Mach number of 3.0, and Reynolds number of $1.68 \times 10^{4}$, based on freestream conditions and the distance from the leading edge to the corner. For a severe test, the experiments conducted by Holden and Moselle 6 are selected for comparison at Mach number 14.1 and Reynolds number $1.04 \times 10^{5}$, with wedge angles of $15^{\circ}$, $18^{\circ}$, and $24^{\circ}$. For the large-wedge-angle case, the pressure rises by a factor of 50 across the interaction. Good agreement is obtained between the present computed results and experimental measurements of wall pressure, skin friction, and heat transfer.

With the whole flowfield calculated, we are able to study the details of the shock wave and viscous flow interaction. The most striking feature is the large, normal static pressure gradients within the boundary layer, which contrasts with the classical boundary-layer assumption. Instead of starting with a local similarity flat plate solution, the generation of a leading edge shock provides another interesting feature. For the experimental cases of Holden and Moselle ${ }^{6}$ we observed from the calculated flowfields that the interference of the leading edge shock with the induced shock significantly affected the pressure and heat transfer distributions.

\section{Analysis}

\section{Governing Fqualions}

The time-dependent Navier-Stokes equations, in two dimensions, neglecting body forces and heat sources, may be written in integral form as

$$
\frac{\partial}{\partial t} \int_{\text {volume }} U d \mathrm{Vol}+\int_{\text {surface }} \bar{H} \cdot \bar{n} d S=0
$$

where

$$
U=\left[\begin{array}{c}
\rho \\
\rho u \\
\rho v \\
E
\end{array}\right) \quad \bar{H}=\left(\begin{array}{l}
\rho \bar{q} \\
\rho u \bar{q}+\overline{\bar{\tau}} \cdot \bar{e}_{x} \\
\rho v \bar{q}+\overline{\bar{\tau}} \cdot \bar{e}_{y} \\
E \vec{q}+\overline{\bar{\tau}} \cdot \bar{q}-K \nabla T
\end{array}\right)
$$

where

$$
\begin{aligned}
& F=\left\{\begin{array}{l}
\rho u \\
\rho u^{2}+ \\
\rho u v+ \\
E u-
\end{array}\right. \\
& \mu\left(\frac{\partial u}{\partial y}+\frac{\partial v}{\partial x}\right)
\end{aligned}
$$

and $\vec{e}_{1}, \bar{e}_{1}$ are unit vectors of the orthogonal $x, y$ coordinate system, and $\bar{n}$ a unit normal vector of the surface enclosing

$$
\begin{aligned}
& \bar{q}=u \vec{e}_{x}+v \bar{e}_{y} \quad E=\rho\left(e+1 / 2\left(u^{2}+v^{2}\right)\right) \\
& \overline{\bar{\tau}}=\sigma_{s} \vec{e}_{x} \vec{e}_{x}+\tau_{x y} \bar{e}_{x} \vec{e}_{y}+\tau_{y} \bar{e}_{y} \vec{e}_{x}+\sigma_{y} \bar{e}_{y} \bar{e}_{y} \\
& \sigma_{x}=p+2 / 3 \mu\left(\frac{\partial u}{\partial x}+\frac{\partial v}{\partial y}\right)-2 \mu \frac{\partial u}{\partial x}, \tau_{x y}=\tau_{y x}=-\mu\left(\frac{\partial u}{\partial y}+\frac{\partial v}{\partial x}\right) \\
& \sigma_{y}=p+2 / 3 \mu\left(\frac{\partial u}{\partial x}+\frac{\partial v}{\partial y}\right)-2 \mu \frac{\partial v}{\partial y}, \nabla T=\frac{\partial T}{\partial x} \vec{e}_{x}+\frac{\partial T}{\partial y} \vec{e}_{y}
\end{aligned}
$$

the volume of integration. The equation of state relates the pressure $p$ and density $\rho$ to temperature $T$ and specific internal energy $e$. The perfect gas relations are $p=\rho R T$ and $e=C_{t} T$. The viscosity coefficient $\mu$ is assumed a function of temperature only, and is evaluated by Sutherland's semiempirical formula $\mu=2.270 \times 10^{-8} T^{3 / 2} /(T+198.6)$ (slug/ft-sec). For the conditions of the experiment and the calculated temperature distribution we assume that the above relations are adequate for this study. The coefficient of thermal conductivity $K$ is computed by assuming a constant Prandtl number $\operatorname{Pr}$ and specific heat $c_{i}$, so that $K={ }_{\gamma} C_{i} \mu / \operatorname{Pr}$.

\section{Time-Split Numerical Technique}

A time-split, two-step MacCormack scheme is used which solves the set of two-dimensional equations by reducing them to two sets of one-dimensional equations. The system of equations can be solved in one orthogonal $x, y$ coordinate system for an arbitrary quadrilateral volume element, as described by Deiwert. ${ }^{12}$ The typical element used is shown in Fig. 2, where $\theta$ is the inclination of the parallelogram element with respect to the $x$-coordinate. The difference equations approximating Eq. (I) are then written as follows

\section{$L x(\Delta t)$ operator:}

$$
\frac{\text { predictor }}{U_{i, j}^{n+1 / 2}}=U_{i, j}^{n}-\frac{\Delta t}{\mathrm{Vol}_{i, j}}\left[\left(F_{i, j}^{n}-F_{i-l, j}^{n}\right) \cdot \Delta y_{j}\right]
$$

$$
\begin{aligned}
& \text { corrector } \\
& \begin{aligned}
U_{i, j}^{n+1 / 2}=1 / 2 & {\left[\overline{U_{i, j}^{n+1 / 2}}+U_{i, j}^{n}-\frac{\Delta t}{\mathrm{Vol}_{i, j}} \overline{\left(F_{i+1, j}^{n+1 / 2}\right.}\right.} \\
& -\overline{\left.\left.F_{i, j}^{n+1 / 2}\right) \cdot \Delta y_{j}\right]}
\end{aligned}
\end{aligned}
$$

\section{$L y(\Delta t)$ operator:}

predictor

$$
\begin{aligned}
& \overline{U_{i, j}^{n+} \leq} U_{i, j}^{n+1 / 2}-\frac{\Delta t}{\mathrm{VoI}_{i, j}}\left[\left(G_{i, j}^{n+1 / 2}-G_{i, j-I}^{n+1 / 2}\right) \cdot \Delta x_{i}\right. \\
& \left.-\left(F_{i, j}^{n+1 / 2}-F_{i, j-l}^{n+1 / 2}\right) \cdot \Delta x_{i} \cdot \tan \theta\right]
\end{aligned}
$$

$$
\begin{aligned}
& \text { corrector } \\
& U_{i, j}^{n+1}=1 / 2\left\{\overline{U_{i, j}^{n+1}+} U_{i, j}^{n+1 / 2}-\frac{\Delta l}{\mathrm{Vol}_{i, j}}\left[\overline{\left(G_{i, j+1}^{n+I}\right.}-\overline{G_{i, j}^{n+1}}\right) \cdot \Delta x_{i}\right. \\
& -\left(\overline{\left.\left.F_{i, j+1}^{n+1}-\overline{\left.F_{i, j}^{n+l}\right)} \cdot \Delta x_{i} \cdot \tan \theta_{i}\right]\right\}}\right.
\end{aligned}
$$

The subscripts $i, j$ refer to a special mesh of point $x_{i}, y_{j}$ with spacing $\Delta x_{i}$ and $\Delta y_{j}$, and the superscript $n$ refers to times $t=n \Delta t$ where $\Delta t$ is the time increment that the solution is advanced. The bar on $\bar{F}$ and $\bar{G}$ indicates that the predicted quantities $\bar{U}$ are to be used in the evaluation of these terms. Note that the operator $L x$ accounts for the convection and stress terms acting at the vertical sides of the typical mesh element shown in Fig. 2 and the Ly operator accounts for the terms acting on the inclined sides. The dot product of Eq. (1) is $F \Delta y_{j}$ for a vertical side and $\left(G+F \tan \theta_{i}\right) \Delta x_{i}$ for an inclined side. The $L x$ and $L y$ operators are applied so that Eq. (1) is approximated at each time-step for each element of the non- 


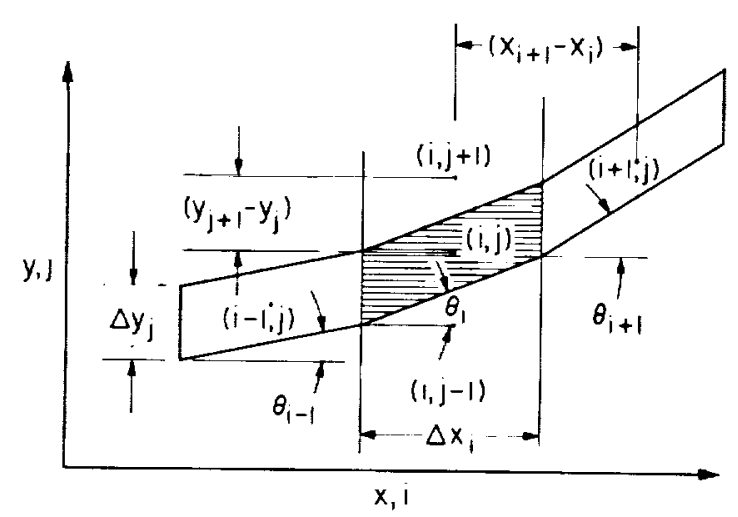

Fig. 2 Parallelogram volume element used in computation.

orthogonal computational mesh. Although the operator sequence of $L y(\Delta t) L x(\Delta t)$ is accurate only to the first order, MacCormack has shown that symmetrical operator sequences such as

$$
L x\left(\frac{\Delta t}{2}\right) L y(\Delta t) L x\left(\frac{\Delta t}{2}\right)
$$

are accurate to the second order both in time and space.

To evaluate the viscous and heat conduction derivatives for the nonorthogonal cell (Fig. 2), the differences used are as follows: Using $\phi$ as a dummy variable, for the $L x$ operator predictor

$$
\begin{aligned}
& \left(\frac{\partial \phi}{\partial x}\right)_{i, j 3} \approx \frac{\phi_{i+1, j}-\phi_{i, j}}{x_{i+1}-x_{i}}-A_{i, j} \frac{\phi_{i, j+1}-\phi_{i, j-1}}{y_{j+1}-y_{j-1}} \\
& \left(\frac{\partial \phi}{\partial y}\right)_{i, j} \approx \frac{\phi_{i, j+1}-\phi_{i, j-1}}{y_{j+1}-y_{j-1}}
\end{aligned}
$$

and for the $L y$ operator predictor

$$
\begin{aligned}
& \left(\frac{\partial \phi}{\partial x}\right)_{i, j} \approx \frac{\phi_{i+1, j}-\phi_{i-1, j}}{x_{i+1}-x_{i-1}}-B_{i, j} \frac{\phi_{i, j+1}-\phi_{i, j}}{y_{j+1}-y_{j}} \\
& \left(\frac{\partial \phi}{\partial y}\right)_{i, j} \approx \frac{\phi_{i, j+1}-\phi_{i, j}}{y_{j+1}-y_{j}}
\end{aligned}
$$

where

$$
\begin{aligned}
& A_{i, j}=1 / 2\left(\tan \theta_{i+1}+\tan \theta_{i}\right) \\
& B_{i, j}=1 / 4\left(\tan \theta_{i+1}+2 \tan \theta_{i}+\tan \theta_{i-1}\right)
\end{aligned}
$$

Similar approximations are used in the corrector steps, such that the overall treatment of derivatives results in centered second-order-accurate approximations. For variable mesh

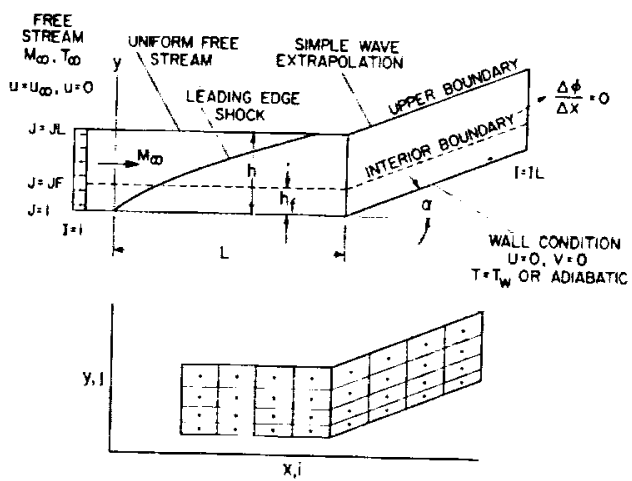

Fig. 3 Computational boundaries (above), and mesh point and cell system (below).

Reynolds number is computed by $(\Delta y)_{\text {min }}=2 / 3\left(L \sqrt{R e_{l}}\right)$. The leading edge is placed at $x=0$ and $L$, the distance from the leading edge to the corner, is used as the characteristic length.

The appropriate boundary conditions are shown in Fig. 3. At the upstream boundary a few mesh points ahead of the leading edge, the flow is assumed uniform at supersonic freestream conditions $\left(M_{\infty}, T_{\infty}, p_{\infty}, u_{\infty}\right)$. The downstream boundary is positioned far enough from the corner so that all the gradients in the flow direction can be set to zero. Though this condition is not exact, the boundary layer in the vicinity of this exit is parabolic, and the remainder of the flow is supersonic; hence, it is not expected that this condition will introduce significant error in the region of interest upstream. The upper boundary is specified by the freestream conditions ahead of the leading edge shock and by simple wave extrapolation downstream of the shock. The wall surface is assumed impermeable, and nonslip boundary conditions are applied. The wall is treated either as isothermal or adiabatic, and the wall pressure is evaluated from an approximation of the $y$-momentum equation at the wall. During the calculation of the inner mesh, transport and stress at the internal boundary $y=h_{f}$ are saved, and their average net quantities are then used as boundary conditions for the outer mesh flow field.

\section{Computational Time-Step}

The maximum time-step for which the calculation will be stable is determined by the CFL and viscous stability requirement. By applying a von Neumann stability analysis separately to the linearized inviscid and viscous parts of Eqs. (1)" we arrive at the following criteria: For the $\operatorname{Lx}\left(\Delta t_{x}\right)$
operator

$$
\Delta t_{x} \leq\left\{\Delta x /\left[|u|+a+\frac{l}{\rho}\left(\frac{2 K}{\Delta x}+\frac{\mu}{\Delta y_{j}}\right)\right]\right\}_{\min }
$$

and for the $L y\left(\Delta t_{y}\right)$ operator

$$
\Delta t_{y} \leq\left\{\Delta y_{j} /\left[\left|v-u \tan \theta_{i}\right|+a \sec \theta_{i}+\frac{l}{\rho}\left(\frac{2 K}{\Delta y_{j}}+\frac{\mu}{\Delta x}\right)\right]\right\}_{\text {min }}
$$

spacing the resulting difference equations are second-order accurate in the computational coordinate system. ${ }^{13}$

\section{Mesh and Boundary Conditions}

Figure 3 shows the computational domain and the mesh point and cell system used for calculation. Note that the inclination angle $\theta$ is zero on the flat plate and is constant along the wedge. The mesh is equally spaced in the $x$-direction, but the $y$-direction, a fine, exponentially stretched mesh spacing is used in the region $0.0<y<h_{f}$ near the wall for resolving the viscous layer, and a coarse, equally spaced mesh is used in the outer region $h_{f}<y<h$, where viscous effects are negligible. The minimum spacing for the fine mesh is dependent upon the where $a$ is the local sound speed. These stable time-steps are larger than those of explicit finite difference methods without time splitting. Four different time-steps are used in calculations: two for the $L x$ operator $\Delta t_{x c}, \Delta t_{x y}$ and two for the $L y$ operator $\Delta t_{y c}, \Delta t_{y f}$ in the coarse and fine mesh, respectively. In general, for the present calculations $\Delta t_{y f}>\Delta t_{y f}$ and $\Delta t_{y c}>\Delta t_{x i}$. Hence, the computational efficiency is enhanced by use of the operator sequences for the fine mesh region

$$
\left[L y\left(\Delta t_{f} / 2\right) L x\left(\Delta t_{f}\right) L y\left(\Delta t_{f} 2\right)\right]^{m}
$$

and for the coarse mesh region

$$
\left[L x\left(\Delta t_{c}\right) L y\left(2 \Delta t_{c}\right) L x\left(\Delta t_{c}\right)\right]
$$


where

$$
\begin{gathered}
\Delta t_{f}=\min \left[\Delta t_{x f}, 2 \Delta t_{y f}\right] \\
\Delta t_{c}=m \cdot \Delta t_{f} \leq \min \left[\Delta t_{x c}, 1 / 2 \Delta t_{y c}\right]
\end{gathered}
$$

and $m$ is an integer, representing the number of applications of the operator sequence in the inner mesh region for each time-step $\Delta t_{c}$ which the solution is advanced in the outer region. As $m$ becomes large, most of the computing time is spent in the calculation of the fine mesh-region.

\section{Nonlinear Instability}

Several types of nonlinear instability are encountered in the present calculation. The remedies for these have been previously discussed by MacCormack, ${ }^{10}$ Baldwin and MacCormack, ${ }^{14}$ and Kutler, Sakell, and Aiello. ${ }^{\text {is }}$ One of them occurs, for example, in the convective flux across a mesh surface when there is an expansion in which the velocity normal to the mesh surface changes sign. When this condition occurs, instability can be avoided by employing the average normal velocity. Another nonlinear instability can be removed by adding a product fourth-order damping term $\Delta U_{i, j}$ to the righthand side of the predictor and corrector equation of each operator. For the $L x$ operator

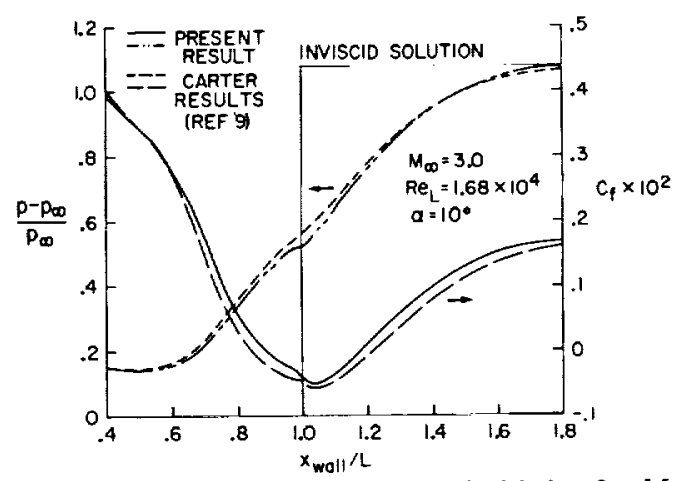

Fig. 4 Comparison of wall pressure and skin friction for $M_{\infty}=3.0$, $\operatorname{Re}_{L}=1.68 \times 10^{4}, \alpha=10^{\circ}$.

pressure, skin friction, and heat transfer along the wall. The flow conditions selected are as follows:

$$
\begin{array}{ll}
M_{\infty}=14.1 & \operatorname{Re}_{L}=1.04 \times 10^{5} \quad \operatorname{Pr}=0.72 \\
T_{\infty}=130^{\circ} \mathrm{R} & L=1.44 \mathrm{ft} \quad T_{w}=535^{\circ} \mathrm{R}
\end{array}
$$

The wall is highly cooled, so that the ratio of wall temperature $T_{w}$ to the adiabatic wall temperature $T_{a w}$ is 0.118 . Note that,

$$
\Delta U_{i, j}=C_{x} \frac{\left|p_{i+l, j}-2 p_{i, j}+p_{i-1, j}\right|\left(U_{i+l, j}-2 U_{i, j}+U_{i-l, j}\right)\left(\left|u_{i, j}\right|+a_{i, j}\right) \Delta t}{\left(p_{i+l, j}+2 p_{i, j}+p_{i-l, j}\right) \Delta x}
$$

and for the $L y$ operator

$$
\Delta U_{i, j}=C_{y} \frac{\left|p_{i, j+1}-2 p_{i, j}+p_{i, j-1}\right|}{\frac{\left(U_{i, j+1}-2 U_{i, j}+U_{i, j-1}\right)\left(\left|v_{i, j}\right|+a_{i, j}\right) \Delta t}{\left(p_{i, j+1}+2 p_{i, j}+p_{i, j-1}\right) \Delta y_{j}}}
$$

where $C_{x}$ and $C_{y}$ are two assigned constants with values between 0.0 and 0.5 . The damping form of the damping term is more compact than standard fourth-order smoothing terms. It requires data at just three mesh points instead of the usual five points. For shock wave calculations this compactness achieves better shock resolution. In the present investigation these terms played important roles in smoothing the ripples in the solution in the region near the leading edge and corner shocks. With this smoothing treatment, fine-mesh spacing near the leading edge, such as that found necessary by Carter ${ }^{9}$ as well as others, in the $x$-direction is avoided. This reduces considerably the computation time of the present method from that required by the other investigators. Even more important, high Mach number cases with strong shocks can be solved without difficulty.

\section{Results and Comparisons}

The first case computed was for $M_{\infty}=3.0$ supersonic flow over a $10^{\circ}$ wedge with an adiabatic wall. The flow conditions correspond identically to one of the cases studied by Carter, ${ }^{9}$ and are given as follows:

$$
\begin{aligned}
& M_{\infty}=3.0 \quad \operatorname{Re}_{L}=1.68 \times 10^{4} \quad \alpha=10^{\circ} \\
& T_{\infty}=390 \mathrm{R} \quad L=2.4 \mathrm{in} . \quad T_{w}=T_{\infty}\left[I+(\gamma-I / 2) M_{\infty}^{2}\right)
\end{aligned}
$$

Here, $T_{w}$, the wall temperature, is assumed equal to the freestream stagnation temperature. For this case 86 mesh points were equally spaced in the $x$-direction, and 28 mesh points were used in the $y$-direction, 15 for the inner mesh and 13 for the outer mesh. Figure 4 shows the present computed surface pressure and skin friction distribution. The skin friction coefficient is defined as $c_{f}=\left(\tau_{w} / \rho_{\infty} u_{\infty}^{2}\right)$. These distributions are in good agreement with the results of Carter.

The experiments selected for comparison were conducted by Holden and Moselle. ${ }^{6}$ Measurements were made of in this case, both the Mach number and Reynolds number are almost one order of magnitude higher than for the previous calculation. The computational domain extends from $(x / L)$ $=-0.077$ to $(x / L)=1.859$, with the fine-mesh boundary placed at $h_{f}=0.096 \mathrm{ft}$ and the outer boundary at $h=0.28 \mathrm{ft}$. In the $x$-direction 90 mesh points were used with a mesh spacing of $\Delta x=3.130 \times 10^{-2} \mathrm{ft}$. In the $y$-direction $30 \mathrm{mesh}$ points 20 for the fine mesh and 10 for the coarse mesh, were used. Mesh spacing varied from $\Delta y=2.087 \times 10^{-3} \mathrm{ft}$ to $\Delta y$ $=9.842 \times 10^{-3} \mathrm{ft}$ for the fine mesh; for the coarse mesh, equal spacing of $\Delta y=2.024 \times 10^{-2} \mathrm{ft}$ was used. In general, the stable time-steps were about $\Delta t_{y f}=3.297 \times 10^{-3} \mathrm{sec}, \Delta t_{x f}$ $=3.216 \times 10^{-6} \mathrm{sec}, \Delta t_{x c}=3.272 \times 10^{-6} \mathrm{sec}, \Delta t_{y c}=1.300 \times$ $10^{-5} \mathrm{sec}$, and hence, $m=10$. The freestream velocity was $u_{\infty}=7.880 \times 10^{3} \mathrm{fps}$, and it took about 200 time-steps (or 1.3 $\mathrm{msec}$ ) to reach a steady state. During this time the freestream traveled a distance seven times the length of the flat plate. About 32 min on a CDC 7600 were required to perform the calculations.

The calculation was first made for a plate plate. Shown for comparison in Fig. 5 is the wall pressure distribution of the

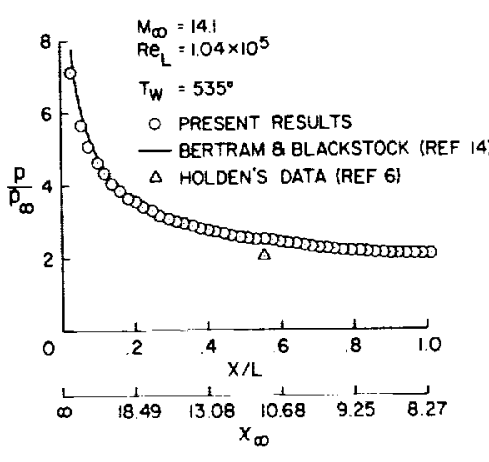

Fig. 5 Comparison of strong interaction theory with present results for wall pressure distribution on flat plate. 

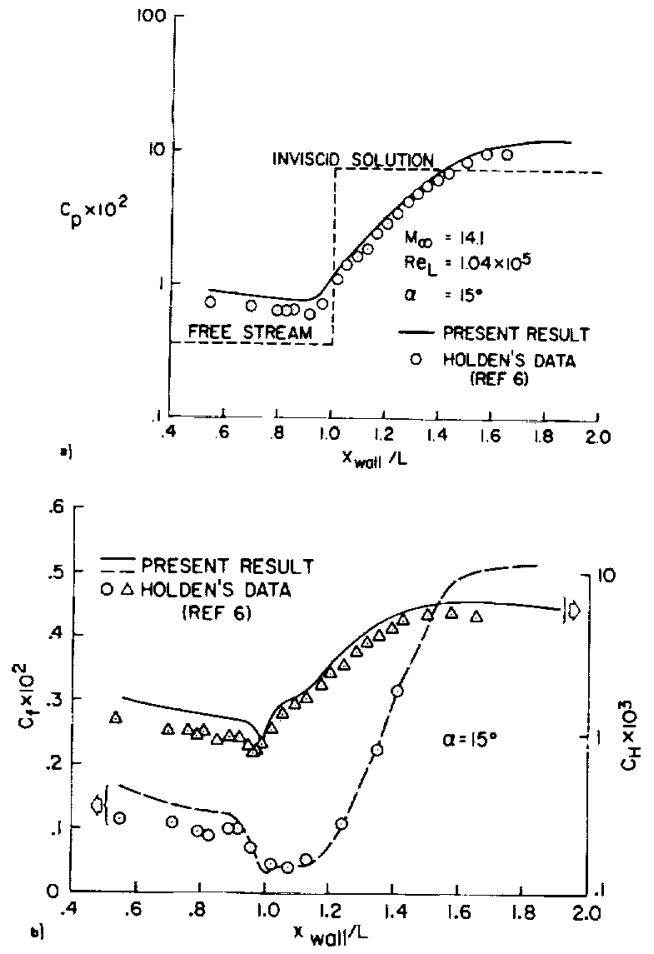

Fig. 6 Comparisons of: a) wall pressure and b) heat transfer and skin friction along wall for $\alpha=15^{\circ}$.
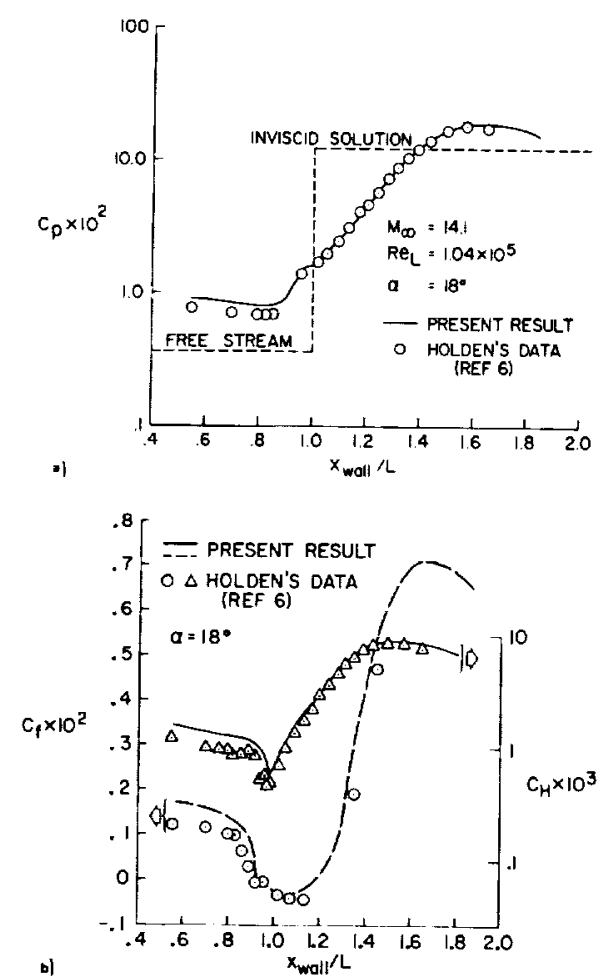

Fig. 7 Comparisons of : a) pressure and b) heat transfer and skin friction along wall for $\alpha=18^{\circ}$.

present computed result and the result theoretically predicted by the strong-interaction analysis of Bertram and Blackstock. ${ }^{16}$ The analysis was based on the hypersonic similarity theory that

$$
p / p_{\infty}=0.83+3 / 4(\gamma(\gamma-1) / 2)^{1 / 2} \beta \chi_{\infty}
$$

where

$$
\beta=1.648 \frac{\gamma-1}{2}\left|\frac{T_{w}}{T_{a w}}+0.352\right|, \quad \chi_{\infty}=\frac{M_{\alpha}^{3} \sqrt{C}}{\sqrt{\operatorname{Re}_{x}}}
$$
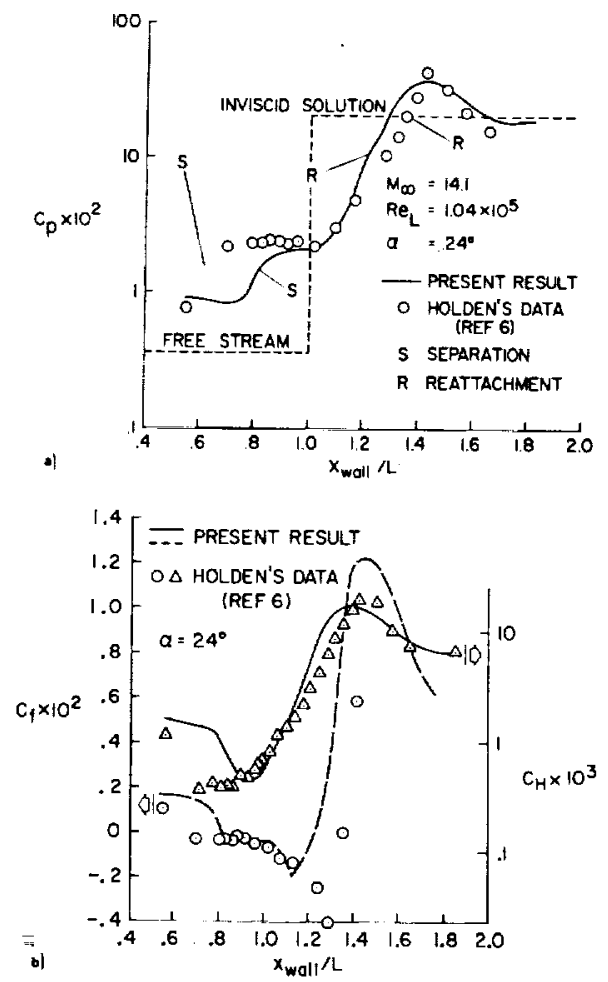

Fig. 8 Comparisons of : a) pressure and b) heat transfer and skin friction for $\alpha=24^{\circ}$.

Here, $\chi_{\infty}$ is a hypersonic parameter; $\beta$ is a parameter related to the wall temperature; and $C$, the Chapman-Rubesin constant, is evaluated as $C=T_{\infty} \mu\left(T_{w^{\prime}}\right) / T_{w} \mu\left(T_{\infty}\right)$. Excellent agreement is obtained between the theoretical prediction and the numerical result. Also shown in Fig. 5 is an experimental value of Holden and Moselle. ${ }^{6}$ The reason for the disagreement between the present result and the experiment is unknown. Nevertheless, as will be seen later, good agreement was observed downstream in the interaction region.

Figures 6-8 show the detailed comparisons of the present computed results with the experimental measurements for pressure, heat transfer, and skin friction for three different wedge angles. Very good agreement was found for the cases of $\alpha=15^{\circ}$ and $\alpha=18^{\circ}$. Here, the pressure coefficient is defined as $c_{p}=\left[p_{\text {wall }} /(1 / 2) \rho_{\infty} u_{\infty}^{2}\right]$ and the heat-transfer coefficient as

$$
c_{H}=\frac{K \frac{\partial T}{\partial y} \sec \theta_{i}}{\rho_{\infty} u_{\infty}\left[\left(e+\frac{p}{\rho}+\frac{u^{2}}{2}\right)_{\infty}-\left(e+\frac{p}{\rho}\right)_{w}\right]}
$$

For $\alpha=15^{\circ}$ there is no separation, while for $\alpha=18^{\circ}$, the separation and reattachment are very close to the experiment. The general features of the computed results for the $\alpha=24^{\circ}$ case have the correct trend but the extent of separation is slightly underpredicted. Nevertheless, the plateau pressure and the magnitude and location of the peaks of surface pressure and heat transfer are correctly predicted. Note that, in the experiment the peaks of pressure and heat transfer coincide, and in the computed results the peak of heat transfer is ahead but very close to those of surface pressure. This is also observed in the case of $\alpha=18^{\circ}$.

With the entire flowfield calculated, we can examine the details of the interaction between viscous and inviscid flows. Fig. 9 shows the isobar distributions for wedge angles of $15^{\circ}$, $18^{\circ}$, and $24^{\circ}$. One of the most striking features is that the static pressure is neither constant across the boundary layer nor constant along the simple straight characteristic lines, as 

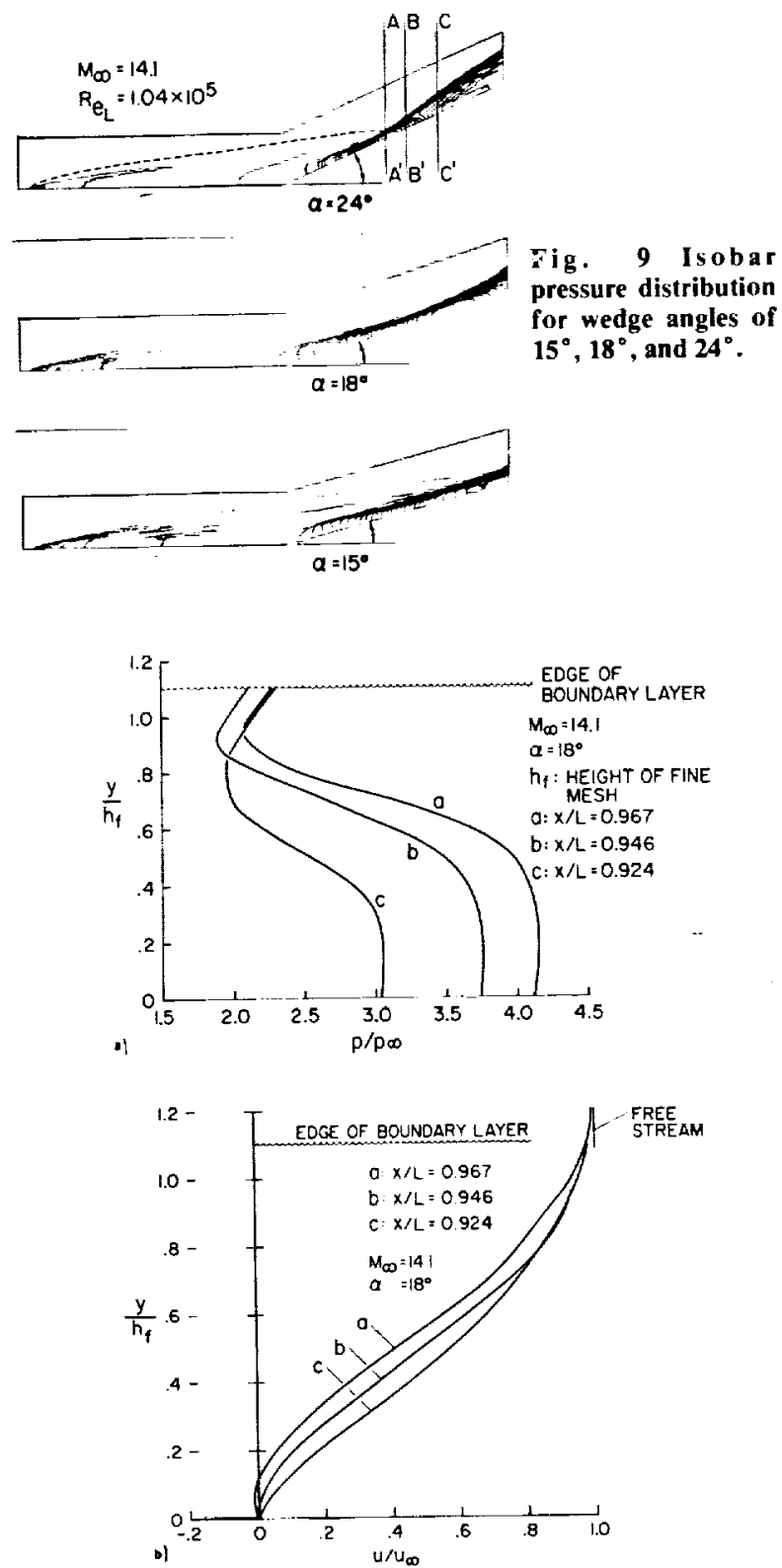

Fig. 10 a) pressure variation and b) $u$-velocity boundary layer profiles near separation for $\alpha=18^{\circ}$.

treated by Myring. ${ }^{17}$ Figure 10 shows the static pressure variation and the corresponding $u$-velocity profiles across the boundary layer at locations near separation for $\alpha=18^{\circ}$. The pressure can be treated as constant only very near the wall, and then it decreases drastically to the edge of the boundary layer. The total variation may be as much as a factor of two or more, depending on the location and wedge angle. Presumably, the normal pressure gradient develops as a result of the curvature of the streamlines in the boundary layer. This curvature can be generated by the response of the boundary layer to the longitudinal pressure gradient or form the curvature of the surface upon which the boundary layer is growing. The pressure rise is first detected near the wall and, because of the large Mach number, takes quite a distance to reach the edge of the boundary layer. The ratio of this distance to the boundary-layer thickness may be very large, and hence, $(\Delta p / \Delta y) \gg(\Delta p / \Delta x)$ may occur.

Downstream of the corner, the compression waves coalesce into a shock wave. The intersection of the leading edge shock with this induced shock shows another interesting feature. Here both the leading edge shock and the induced shock are right-runing waves. The intersection is classified by Edney ${ }^{18}$

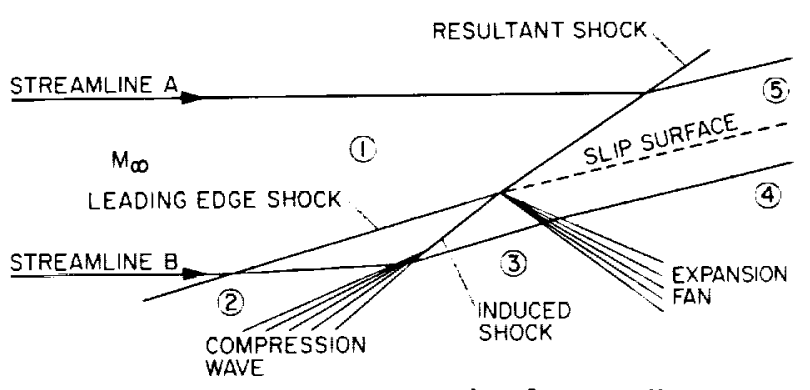

Fig. 11 Type VI shock wave interference pattern.

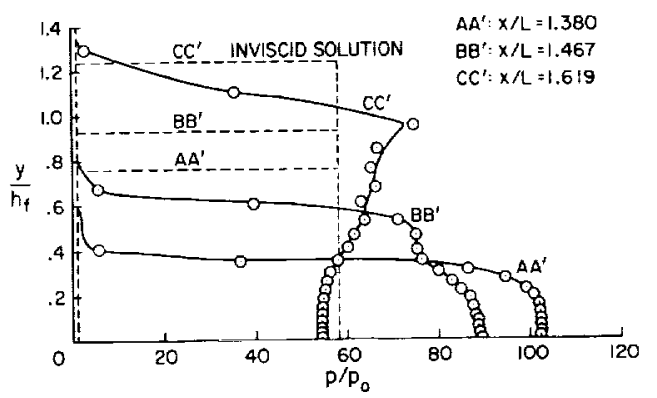

Fig. 12 Pressure profiles at three locations downstream of intersection point of leading edge and induced shocks.

as Type VI interference in which, after the intersection of two shocks, a single stronger resultant shock, and expansion fan, and a slip surface are formed (Fig. 11). Along the streamline $\mathrm{B}$, through multiple compression, the pressure in region 3 is higher than the pressure in region 5, which is compressed along streamline A by a single resultant shock. Behind the expansion fan the pressure and flow direction in region 4 are the same as those in region 5 , which is separated from region 4 by a slip surface. For $\alpha=15^{\circ}$, the expansion fan does not reach the wedge surface within the computational region. The wall pressure monotonically increases and the peak of maximum pressure is expected to lie downstream of the computational domain (Fig. 6a). For $\alpha=18^{\circ}$, the influence of the expansion fan on the wedge surface is observed in the computational domain (Fig. 9); the surface pressure and heat transfer reach their maximum and then decrease gradually (Fig. 7). Since the intersection angle is small, the expansion fan is so weak that it can barely be detected. As the wedge angle increases further to $\alpha=24^{\circ}$, the correlation of the interference of leading edge shock and the peak pressure is evident (Fig. 9). (The dashed line represents, approximately, the trajectory of the leading edge shock.) The high pressure in region 3 (Fig. 11) is attributed to the viscous effect of smoothly bending the streamlines while passing the compression corner, and the high heat-tiansfer rate is attributed to the high temperature and the local thinning of the boundary layer behind the compression waves and the induced shock. The expansion fan causes a rapid decrease in pressure. Figure 12 presents the pressure profiles at three different locations, $\mathrm{AA}^{\prime}, \mathrm{BB}^{\prime}, \mathrm{CC}^{\prime}$ (indicated in Fig. 9), and shows the details of the pressure field change from overcompression, through expansion, to a state of overexpansion relative to the inviscid solution. The expansion fan bounces back and forth, partly transmitted and partly reflected from the viscous layer and slip surface, between the wall and the external resultant shock, and the flow asymptotically relaxes to a state of normal weak interaction. It is interesting to notice that, for a flow at Mach number 14.1 over a $24^{\circ}$ wedge, the pressure rise, $p p_{\infty}$, is 225 for the isentropic solution, and 58 for the inviscid Rankine-Hugoniot relation, whereas $\left(p p_{\infty}\right)_{\max }=102$ for the viscous flow solution in the present calculation.

Indeed there exists no discontinuity or slip surface in the viscous flow; the slip surface between regions 4 and 5 (Fig. 11) is smeared into a shear layer. Figure 13 shows the density con- 


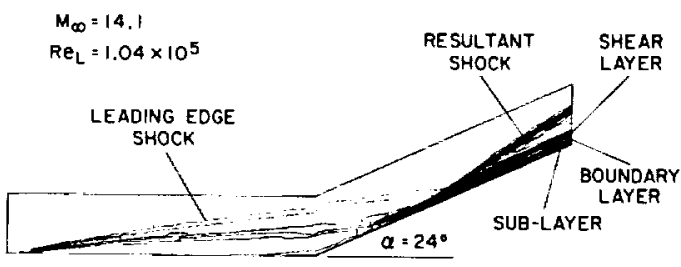

Fig. 13 Density contours and details of shear layer and boundary layer.

Fig. 14 Velocity, tem. perature, and density profiles across boundary layer and shear layer.
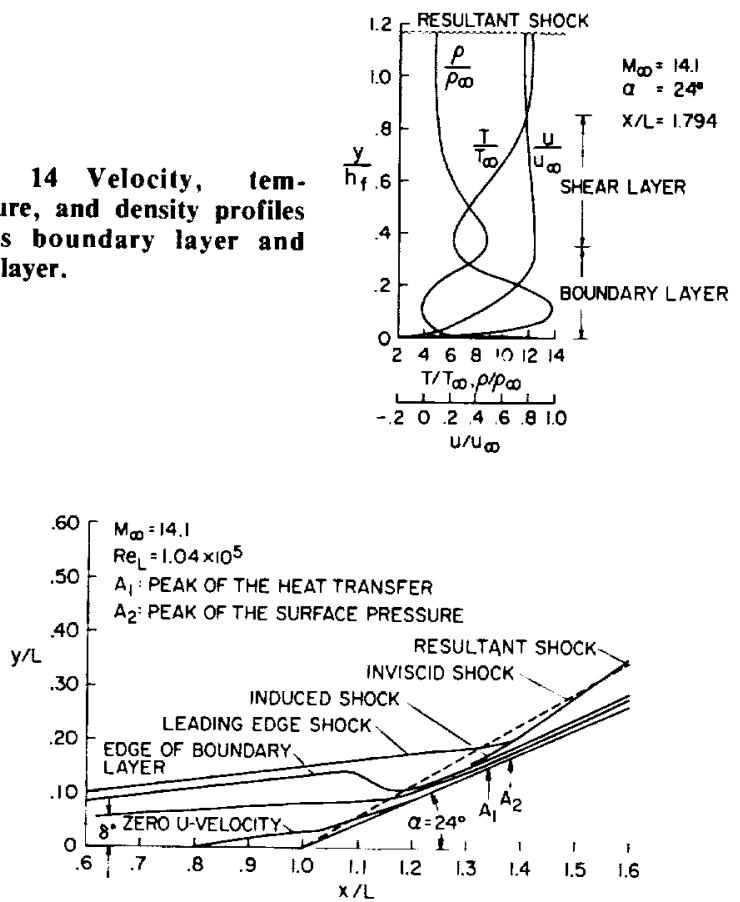

Fig. 15 Locations of Ieading edge shock, induced shock, resultant shock, edge of boundary layer, boundary-layer displacement thickness, and line of zero $u$-velocity for $\alpha=24^{\circ}$.

tours and clearly displays the trajectories of the leading edge shock, resultant shock, shear layer, boundary layer, and the cold-wall high-density sublayer. Figure 14 shows the detailed plots of $u$-velocity, density, and temperature across the boundary layer and the shear layer at the location $(x / L)=179$ for $\alpha=24^{\circ}$. Total pressure loss is larger in region 5 than across the single resultant shock; hence, the temperature is higher, and the velocity and density are lower in region 5 than those in region 4.

Figure 15 indicates the locations of leading edge shock, induced shock, resultant shock, edge of boundary layer, boundary-layer displacement thickness $\delta^{*}$, and the line of zero $u$-velocity for the case of $\alpha=24^{\circ}$. Here, the edge of the boundary layer is defined as the smallest $y$ for which $\Delta(\rho u) / \rho u<0.02$. The locations of the peak heat-transfer rate and the peak wall pressure are labeled $A_{1}$ and $A_{2}$, respectively. The boundary-layer displacement thickness $\delta^{*}$ at first increases as pressure increases, up to the corner, then decreases as pressure continues to increase, reaching a neck region at about the point of peak pressure, and then starts to increase as the normal state of the boundary layer resumes. The thickness of the boundary layer before and after the compression are different by about one order of magnitude.

\section{Conclusion}

An efficient time-splitting finite difference scheme has been used to obtain steady-state solutions of the Navier-Stokes equations for supersonic and hypersonic laminar flows over a compression corner. Favorable comparisons with previous calculation and with experiment indicate that the present calculations are accurate. The pressure profiles are neither constant across the boundary layer nor constant along simple, straight characteristic lines, as has been assumed in some analyses. Surface pressures higher than predicted by inviscid shock wave theory are obtained, due to the compression of the smooth bending of the streamlines. Edney Type VI interference of the leading edge shock with the induced shock results in an expansion fan which produces a large peak in the surface pressure and heat transfer. Consequently, it is important to include the leading edge shock wave in the present study of such hypersonic flows.

\section{References}

${ }^{1}$ Reyhner, T. A and Flügge-Lotz, I., "The Interaction of a Shock Wave with a Laminar Boundary Layer," International Journal of Nonlinear Mechanics, Vol. 3, June 1968, pp. 173-199.

${ }^{2}$ Dwoyer, D. L., "Supersonic and Hypersonic Two-Dimensional Laminar Flow over a Compression Corner," Proceedings of the AIAA Computational Fluid Dynamics Conference, Palm Springs, Calif., July 1973, pp. 69-83.

${ }^{3}$ Lees, L. and Reeves, B. L., "Supersonic Separated and Reattaching Laminar Flows: I. General Theory and Application to Adiabatic Boundary Layer-Shock/Wave Interactions," AIAA Journal, Vol. 2, Nov. 1964, pp. 1907-1920.

${ }^{4}$ Klineberg, J. and Lees, L., "Theory of Laminar Viscous-Inviscid Interaction in Supersonic Flow," AIAA Journal, Vol. 7, Dec. 1969, pp. 2211-2221.

${ }^{5}$ Nielsen, J. N., Goodwin, F. K., and Kuhn, G. D., "Review of the Method of Integral Relations Applied to Viscous Interaction Problems Including Separation," Proceedings of the Symposium on Viscous Interaction Phenomena in Supersonic and Hypersonic Flow, May 1969, Hypersonic Research Lab., Aerospace Research Labs., Wright-Patterson AFB.

${ }^{6}$ Holden, M. S. and Moselle, J. R., "Theoretical and Experimental Studies of the Shock Wave-Boundary Layer Interaction on Compression Surfaces in Hypersonic Flow," CALSPAN Rept. AF-2410A-1, Buffalo, New York, Oct. 1969.

${ }^{7}$ Georgeff, M. P., "Momentum Integral Method for ViscousInviscid Interactions with Arbitrary Wall Cooling," AIAA Journal, Vol. 12, Oct. 1974, pp. 1393-1400.

${ }^{8}$ Van Dyke, M., "Higher-Order Boundary-Layer Theory," Annual Review of Fluid Mechanics, Vol. 1, 1969, Annual Reviews Inc., Palo Alto, Calif., p. 265.

${ }^{9}$ Carter, J. E., "Numerical Solutions of the Navier-Stokes Equations for the Supersonic Laminar Flow over a Two-Dimensional Compression Corner," NASA TR R-385, July 1972.

${ }^{10}$ MacCormack, R. W., "Numerical Solution of the Interaction of a Shock Wave with a Laminar Boundary Layer," Lecture Notes in Physics, Vol. 8, 1972, Springer-Verlag, Berlin, New York, pp. 151163.

"MacCormack, R. W. and Baldwin, B. S., "A Numerical Method for Solving the Navier-Stokes Equations with Application to ShockBoundary Layer," Sandia Labs., Preprint SLA-74-5009 Albuquerque, New Mexico, 1974.

${ }^{12}$ Deiwert, G. S., "Numerical Simulation of High Reynolds Number Transonic Flows," AIAA Paper 74-603, Palo Alto, Calif., 1974.

${ }^{13}$ Blottner, F. G., "Variable Grid Scheme Applicd to Turbulent Boundary Layer," Sandia Labs., Preprint SLA-74-5009, Albuquerque, New Mexico, 1974.

${ }^{14}$ Baldwin, B. S. and MacCormack, R. W., "Interaction of Strong Shock Wave with Turbulent Boundary Layer," AIAA Paper 74-558, Palo Alto, Calif., 1974.

${ }^{15}$ Kutler, P., Sakell, L., and Aiello, G., "On the Shock-on-Shock Interaction Problem," AIAA Paper 74-524, Palo Alto, Calif., June 1974.

${ }^{16}$ Bertram, M. H. and Blackstock, T. A., "Some Simple Solutions to the Problem of Predicting Boundary-Layer Self-Induced Pressure," NASA TN D-798, April 1961.

${ }^{17}$ Myring, D. G., "The Interaction of a Turbulent Boundary Layer and a Shock at Hypersonic Mach Number," AGARD CP-30, 1968.

${ }^{18}$ Edney, B., "Anomalous Heat-Transfer and Pressure Distributions on Blunt Bodies at Hypersonic Speeds in the Presence of an Impinging Shock," FFA Rept. 116, Aeronautical Research Inst. of Sweden, Stockholm, Sweden, Feb. 1968. 

